\title{
Dynamic Responses of Irregular Fibers Under Axial Tension
}

\author{
Wenyi Yan, ${ }^{1}$ Zhi Li, ${ }^{2}$ Xungai Wang, ${ }^{2}$ Lijing Wang ${ }^{3}$ \\ ${ }^{1}$ Department of Mechanical and Aerospace Engineering, Monash University, Clayton, Victoria 3800, Australia \\ ${ }^{2}$ Centre for Material and Fibre Innovation, Deakin University, Geelong, Victoria 3217, Australia \\ ${ }^{3}$ School of Fashion and Textiles, RMIT University, Brunswick, Victoria 3056, Australia
}

Received 1 December 2008; accepted 9 February 2009

DOI 10.1002/app.30219

Published online 28 April 2009 in Wiley InterScience (www.interscience.wiley.com).

\begin{abstract}
A numerical study on the dynamic responses of irregular fibers under axial tension is presented in this article. The irregularity was represented by a sinusoidal shape profile along the fiber axis. The finite element method was used in the simulations and the maximum first principal stress due to the dynamic pulling has been examined. Our numerical results indicate that the first principal stress mainly varies along the longitudinal direction. Its change in radial direction is negligibly small. The maximum first principal stress in an irregular fiber always appears in
\end{abstract}

the narrowest crosssection, which is the weakest link of the fiber. The maximum first principal stress is very sensitive to the change in the irregular amplitude. The stress value increases dramatically with the increase in the amplitude of irregularity. The frequency of irregularity has a limited effect on the maximum first principal stress. () 2009 Wiley Periodicals, Inc. J Appl Polym Sci 113: 2561-2568, 2009

Key words: fibers; modeling; strength; stress; dynamic tensile behavior

\section{INTRODUCTION}

Most natural fibers are inherently nonuniform. Taking wool as an example, the between-fiber and withinfiber diameter variations can be more than $20 \%$. These fibers are subjected to high-speed mechanical actions during processing and fiber nonuniformity is amplified as the fibers are processed at increasingly higher speeds. The most serious consequence of high-speed fiber processing is fiber breakage. In the case of wool fiber, for instance, trials conducted on a typical $21 \mu \mathrm{m}$ wool have shown that about $39 \%$ of the fibers were broken during the carding process, leading to a reduction in a mean fiber length of $28 \mathrm{~mm},{ }^{1}$ which significantly devalued the fiber.

The effect of fiber irregularities on fiber tensile properties has long been recognized, as entailed in the truism that the strength of a chain is that of its weakest link. ${ }^{2}$ Banky and Slen ${ }^{3}$ conducted experiments on irregular wool fibers, and reported a large difference in the amount of extension experienced by segments of the same fiber, with the thinner segments extending more than the thicker segments. Kenny and Chaikin ${ }^{4}$ studied the subject of fiber irregularity analytically. They examined the stressstrain relationships of nonuniform textile materials, au).

Correspondence to: W. Yan (wenyi.yan@eng.monash.edu.

Journal of Applied Polymer Science, Vol. 113, 2561-2568 (2009)

(c) 2009 Wiley Periodicals, Inc. and demonstrated the profound effects of fiber dimensional nonuniformity on fiber stress-strain behavior. For example, they revealed that a typical keratin fiber with a coefficient of variation $(\mathrm{CV})$ of $16 \%$ in crosssection extended $60 \%$ more than a uniform fiber with the same average cross-sectional area, when they were subjected to a particular load. Collins and Chaikin ${ }^{5-7}$ conducted further study in this area. They used an analytical approach to examine the effect of nonuniformity on the tensile properties of nonuniform fibers of special geometries such as a cone. In recent years, there has been a growing awareness of the importance of fiber nonuniformity, particularly the diameter variation of wool. He et al. ${ }^{8}$ used a numerical approach to simulate a wide range of fiber nonuniformities and examined their effect on fiber tensile and flexural behavior. Relationships have been established between the within-fiber diameter variation and the average and maximum strains in a fiber under extension. ${ }^{9,10}$ These reports have unequivocally revealed the significant effect of geometrical nonuniformities on the mechanical behavior of nonuniform fibers. However, their works have focused on the quasi-static mechanical behavior of the nonuniform fibers. Yet almost all stages of fiber processing are dynamic, and the impact velocity can be as high as $30 \mathrm{~m} / \mathrm{s}$. The behavior of these fibers under dynamic loading has a direct impact on fiber breakages and fiber processing efficiency. Despite this, there has not yet been a systematic study on the dynamic behavior of nonuniform fibers. In 
1963, Lyons ${ }^{11}$ summarized the work devoted to the dynamic behavior of fibrous materials, such as relatively uniform continuous filaments and yarns. Geometrical nonuniformity was not taken into consideration. In fact, most of the design criteria for impact items such as parachutes were based on data obtained from quasi-static tests. Minimizing fiber breakage requires a thorough understanding of the mechanical behavior of these nonuniform fibers under dynamic loading conditions, including the stress wave propagation within the nonuniform fibers. There is an obvious gap in the area of understanding the dynamic behavior of nonuniform fibrous materials.

The minimum fiber diameter and the rate of change of diameter along a fiber are important determinants of strength. ${ }^{12}$ A single fiber under tension breaks whenever the force in the fiber produces a local extension at a point along its length that is equal to or greater than the breaking extension of the fiber at that point. Because fibers often vary in cross-sectional area along their lengths, the local extension produced by a tensile force on the fiber will be greatest at the thinnest point; so the chances are that a break will occur at that point. It is difficult to locate the minimum diameter along a fiber because fibers are often elliptical and irregular. Besides, fibers do not always break at the minimum diameter anyway. ${ }^{13}$ Measuring the diameter at the broken ends is frustratingly slow and difficult when the break is not a clean one. Numerical modeling provides an alternative to study the fiber tensile behavior. This article used finite element (FE) technique to model the dynamic responses of irregular fibers under axial tension and revealed the effects of fiber irregularity parameters and pulling speeds on the maximum first principal stress due to the dynamic pulling.

\section{PROBLEM DESCRIPTION}

The physical problem of an irregular fiber under axial dynamic tension is examined in this article. As shown in Figure 1, the fiber with length $L$ is considered as axisymmetric about its longitudinal axis. The irregularity profile along the axis is assumed as a sinusoidal shape. The base radius is $r$ and the amplitude of the variation of the radius is $\delta_{r}$, as illustrated in Figure 1. Therefore, the radius of the profile at any location of $z$ cylindrical coordinate system can be described as:

$$
r(z)=r+\delta_{r} \sin \left(\frac{2 \pi n z}{L}\right),
$$

where $n$ is the number of sinusoidal waves for the fiber and $n=4$ is the case shown in Figure 1. Equa-

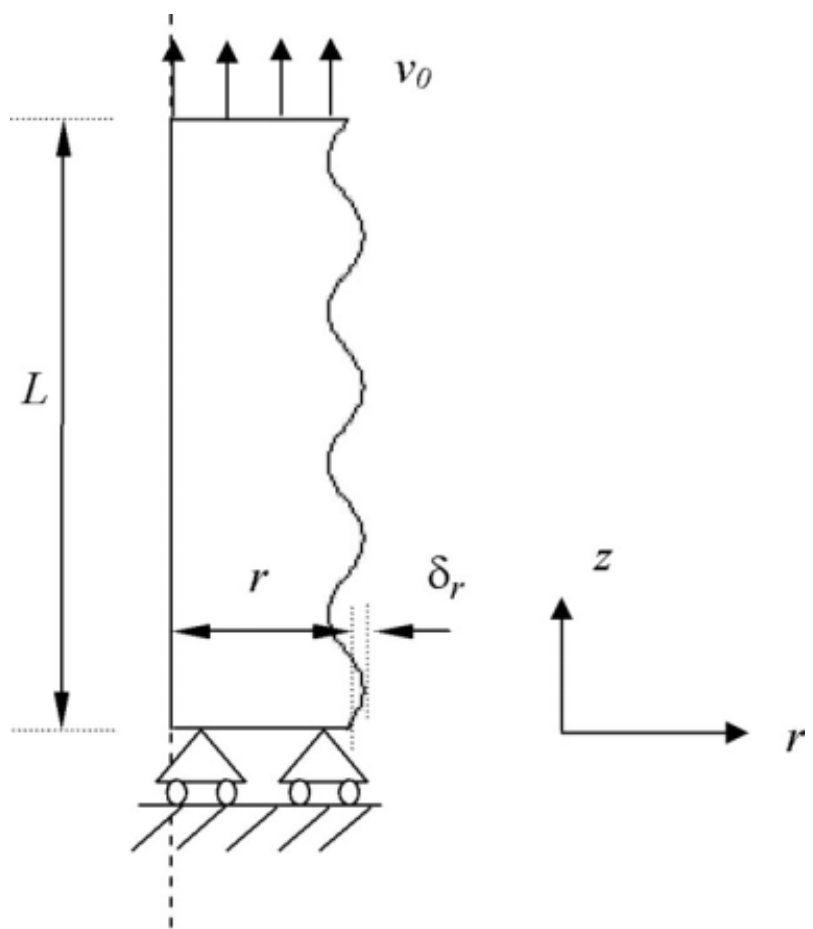

\section{Axisymmetric} axis

Figure 1 Illustration of the axisymmetric section of an irregular fiber under dynamic tension.

tion (1) is applied in the modeling of the geometry in our numerical simulations. In reality, the profile of a real fiber such as wool is very complex. The sinusoidal profile can be treated as an approximation of the real situations. Based on the sine function, the thinnest crosssection, or the weakest link in the fiber, corresponds to the smallest radius $r-\delta_{r}$ and the largest radius $r+\delta_{r}$ represents the thickest crosssection. The value of $\delta_{r}$ is zero for an idealized uniform fiber. The variation of the irregularity along the axis is represented by the parameter $n$. Therefore, such an approximation can be applied to study the effects of fiber irregularity on the fiber dynamic responses.

To simulate the loading process, we consider one end of the fiber is constrained in the axial direction and the other end is pulled along the axial direction with a constant speed $v_{0}$. The total pull distance $\Delta L$ in all our simulations is chosen as $10 \%$ of the fiber length L. Experimental study indicates that some fibers show brittle breakage under dynamic tension. According to the brittle fracture theory, the maximum normal stress, i.e., the first principal stress, $\sigma_{1}$, would determine the breakage at a material point. For a component, the maximum value of the first principal stress in the component, $\sigma_{1}^{\max }$, determines the safety of the component. To investigate the effects of fiber irregularity on its breakage, we calculate the first principal stress $\sigma_{1}$ at any point in the 
fiber during a dynamic loading. From here, we can then calculate the maximum value $\sigma_{1}^{\max }$ in the fiber. Based on our understanding of the physical problem, the value of $\sigma_{1}^{\max }$ should be determined by:

$$
\sigma_{1}^{\max }=f\left(L, r, \delta_{r}, n, v_{0}, E, \mu, \rho\right),
$$

where $E$ is the Young's modulus, $\mu$ is the Poisson's ratio and $\rho$ is the density of the fiber material. Although the stress-strain curves of most fiber materials show a nonlinear behavior under quasi-static loading conditions, a linear behavior is common for fibers under dynamic loading conditions. ${ }^{11}$ Therefore, we simplify the fiber as a linear elastic material in our current study. This simplification can be easily removed once relevant material data are available. In the following simulations, we chose $L=5$ $\mathrm{mm}, r=10 \mu \mathrm{m}, E=3000 \mathrm{MPa}, \mu=0.35$ and $\rho=$ $1.31 \times 10^{3} \mathrm{~kg} / \mathrm{m}^{3}$ and examined the influence of irregularity parameters $\delta_{r}$ and $n$ under different tensile speed $v$ on the maximum normal stress $\sigma_{1}^{\max }$.

\section{FINITE ELEMENT MODEL AND MODEL VERIFICATION}

The FE method was used to numerically study the dynamic responses of irregular fibers under dynamic axial tension. All the simulations were carried out by using the commercial package ABAQUS/ Explicit. ${ }^{14}$ As discussed earlier, axisymmetric models were applied to simulate the physic problems. Very fine mesh with the element side of about $2 \mu \mathrm{m}$ was applied through the axisymmetric sections to ensure the accurate results. Figure 2 shows two examples of the FE mesh of a portion of the simulated fibers. Each FE model contains a total of 12,500 four-node axisymmetric elements.

Elastic wave propagation in a uniform thin bar due to dynamic axial loading is a traditional problem in the study of elastic waves. ${ }^{15,16}$ One of the applications of this study is to develop a dynamic tensile test for ceramics. ${ }^{17}$ For the purpose of verifying our FE model, an idealized uniform fiber with a constant crosssection area is considered. In the theoretical one-dimensional problem, the displacement along the axial direction $u(z, t)$ is governed by the following partial differential equation: ${ }^{11}$

$$
\frac{\partial^{2} u}{\partial t^{2}}=c^{2} \frac{\partial^{2} u}{\partial z^{2}}, \quad c=\sqrt{\frac{E}{\rho}}
$$

where $c$ is the elastic wave speed. The boundary conditions are

$$
u(0, t)=0 \quad \text { and } \quad \frac{\partial u}{\partial t}=v_{0} \quad \text { at } \quad z=L \text { for } t \geq 0 .
$$

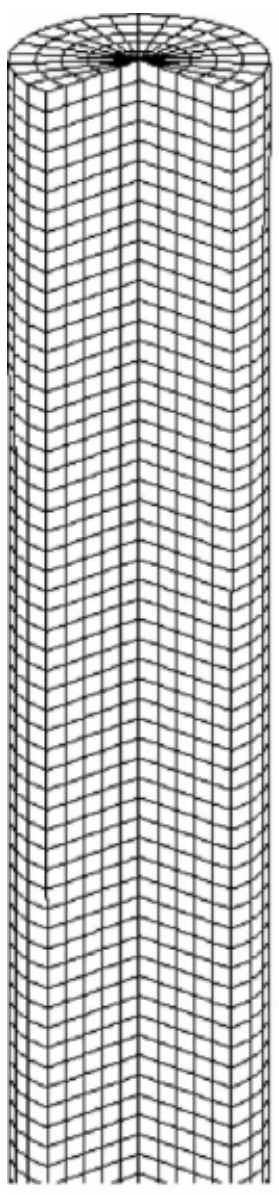

(a)

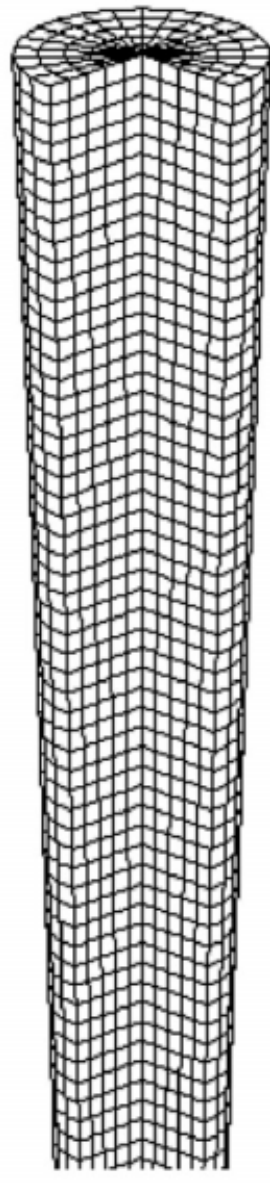

(b)
Figure 2 Examples of a partition of the swept FE models for (a) uniform fiber and (b) irregular fiber with $n=5$ and $\delta_{r}=5 \mathrm{~mm}$.

The initial conditions are

$$
u(z, 0)=0 \quad \text { and } \quad \frac{\partial u}{\partial t}=0 \text { at } t=0 .
$$

The problem of a bar fixed at one end, which is longitudinally hit by a moving body, is solved in text book by Love. ${ }^{15}$ If the striking body has an infinite mass and it pulls the bar, then Love's problem is equivalent to the current one. Therefore, we can derive the analytical solution to this current problem. ${ }^{15}$ For example, the axial stress is determined by:

$$
\begin{array}{r}
\sigma_{\mathrm{zz}}(z, t)=-E\left[f^{\prime}(\xi)+f^{\prime}(\eta)\right] \quad \text { with } \xi=c t-z \\
\text { and } \eta=c t+z
\end{array}
$$

The function $f^{\prime}(\xi)$ or $f^{\prime}(\eta)$ is

$$
f^{\prime}(\zeta)=\left\{\begin{array}{cc}
0 & -L<\zeta<L \\
-v_{0} / c & L<\zeta<3 L \\
-2 v_{0} / c & 3 L<\zeta<5 L \\
-3 v_{0} / c & 5 L<\zeta<7 L \\
\cdots &
\end{array}\right.
$$


where $\zeta$ can be either $\xi$ or $\eta$. Applying eqs. (6) and (7), we can find the axial stress at any point, $\sigma_{\mathrm{zz}}(\mathrm{z}$, $t)$, during the dynamic process. At the fixed end, $z=$ 0 , we have

$$
\sigma_{\mathrm{zz}}(0, t)=\left\{\begin{array}{cc}
0, & -L<\zeta<L \\
2 E v_{0} / c, & L<\zeta<3 L \\
4 E v_{0} / c, & 3 L<\zeta<5 L \\
8 E v_{0} / c, & 5 L<\zeta<7 L \\
\ldots &
\end{array}\right.
$$

The stress value as a function of the time $t$ is shown as the solid curve in Figure 3 for the case $v_{0}$ $=20 \mathrm{~m} / \mathrm{s}$. The stress value was zero at the fixed end until the first stress wave reached at $t=3.31 \mu$ s. The stress wave got reflected at the end and the second stress wave with doubled amplitude then reached the bottom after another $3.31 \mu$ s. This stress wave propagated and got reflected continuously, which formed a step-shape stress versus time curve.

In Figure 3, the analytical solution is compared with two FE results with different Poisson's ratios of $\mu=0.35$ and $\mu=0.05$. It clearly indicates that the one-dimensional analytical solution agrees very well with the FE solutions. Our FE model considered a 3D problem by using axisymmetrical elements. The small difference between the analytical solution and the FE solutions is caused by the 3D effect and the large deformation toward the end of the dynamic process. Reducing the Poisson's ratio of $\mu$ can reduce the 3D effect. Consequently, the FE curve for $\mu=$ 0.05 is closer to the one-dimensional analytical curve. The one-dimensional analytical solution eq. (8) is based on small deformation theory. The deformation of the fiber increases with the impact time. Toward the end of the dynamic process, for example, the elongation $\Delta L / L=10 \%$ at $t=25 \mu \mathrm{s}$, the strain based on large deformation theory should be applied. This explains why the difference between the analytical solution based on small deformation theory and the FE solution based on large deformation theory increases with the impact time $t$. Considering the 3D effect, the irregularity and the large deformation involved in the dynamic pulling, the numerical FE method is needed to investigate this problem. The FE results from the uniform fiber with $\mu=0.35$ have been used as the reference to examine the irregularity effect in this study.

It is worth pointing out that the value of the maximum value of $\sigma_{\mathrm{zz}}$, which is roughly the maximum first principal stress, could be far more greater than the breaking stress of a fiber. As the purpose of this study is to examine the dynamic response of irregular fibers not the breaking stress of irregular fibers, it is the relative stress values, rather than the absolute, that are of interest. Additionally, we are interested in the maximum first principal stress $\sigma_{1}^{\max }$ in the

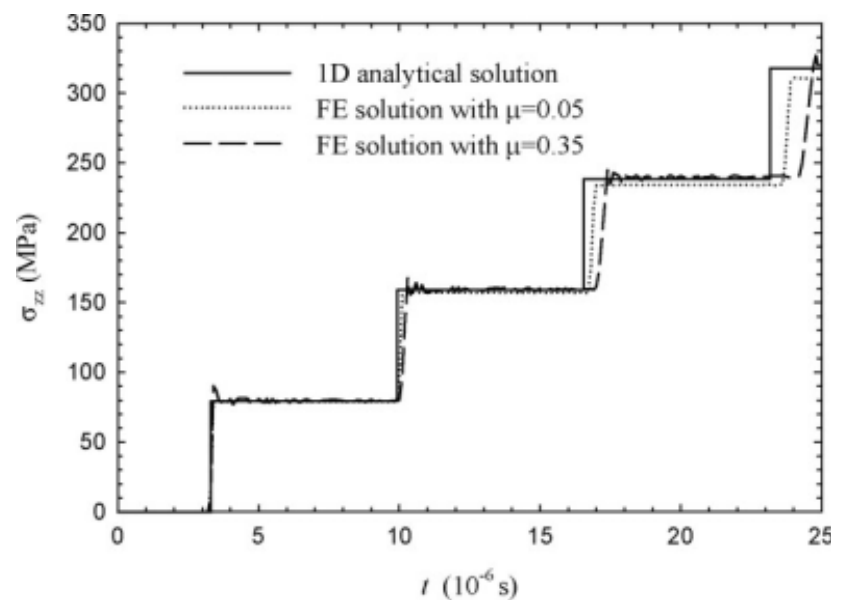

Figure 3 One-dimensional analytical solution of the axial stress at the fixed end, compared with two FE solutions for $\mu=0.05$ and $\mu=0.35$, respectively.

fiber under dynamic tension, whose value determines the breakage of the fiber.

\section{RESULTS AND DISCUSSION}

In addition to the material properties, $E, \mu$, and $\rho$ and the basic geometrical parameters, $L$ and $r$ in eq. (2), the $\sigma_{1}^{\max }$ depends on the irregularity parameters, $\delta_{r}$ and $n$, and the pulling speed, $v_{0}$. To consider the irregularity effects, the results of the influence of $\delta_{r}$, $n$ under different $v_{0}$ on the maximum normal stress from our FE simulations are discussed later.

\section{Effects of irregularity radius amplitude $\boldsymbol{\delta}_{r}$}

The first principal stress $\sigma_{1}$ at any point in a fiber during a dynamic pulling process can be obtained from our FE simulation. Under the same pulling speed of $v_{0}=20 \mathrm{~m} / \mathrm{s}$ and the same irregular frequency of $n=5$, Figure 4 shows the contour plots of this variable for the fibers with different irregularity radius amplitudes $\delta_{r}$. Case (a) indicates that the maximum value of the maximum normal stress appeared at the fixed end of the regular uniform fiber. Cases (b)-(f) correspond to 5 irregular fibers with $\delta_{r}$ varying from 1-5 $\mu \mathrm{m}$. Comparing these contours and observing Figures 5 and 6, one can obtain the following:

1. The maximum first principal stress $\sigma_{1}^{\max }$ in an irregular fiber always appears in one of the narrowest crosssections, which is the weakest link of the fiber. As the fixed fiber end does not correspond to one of the narrowest crosssections, this maximum value does not appear at the bottom $(z=0)$, which is completely different from that of the uniform fiber. Further more, the 


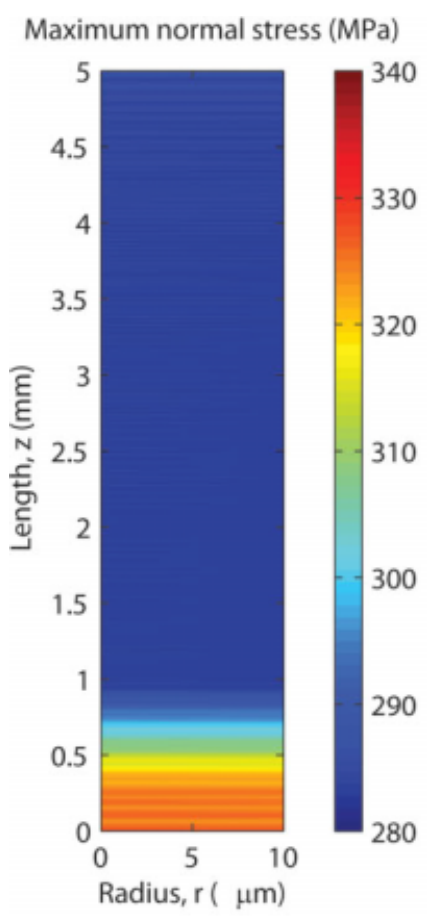

(a)

Maximum normal stress ( $\mathrm{MPa}$ )

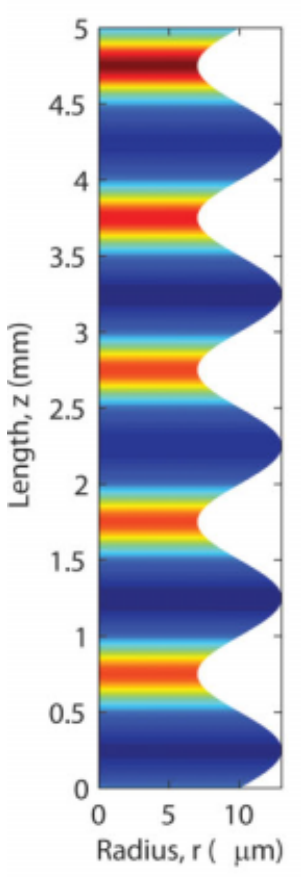

(d)

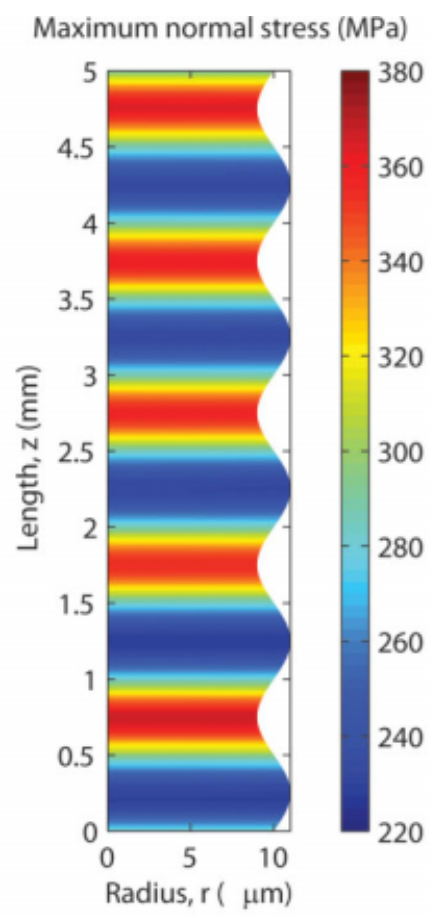

(b)

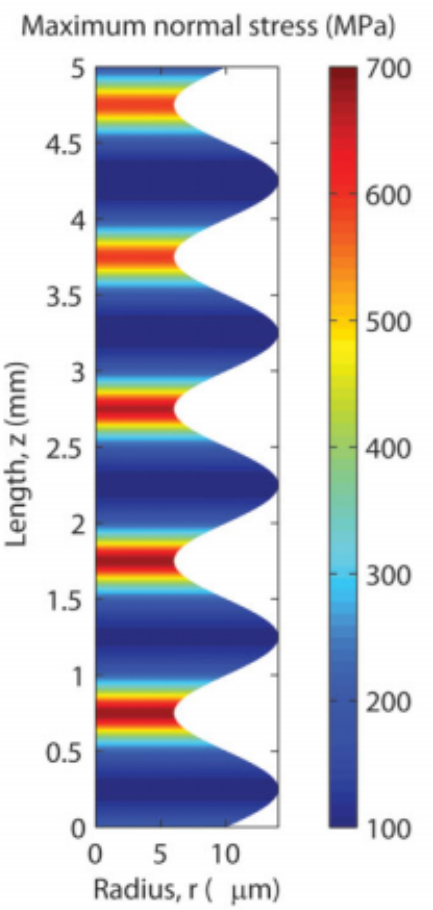

(e)

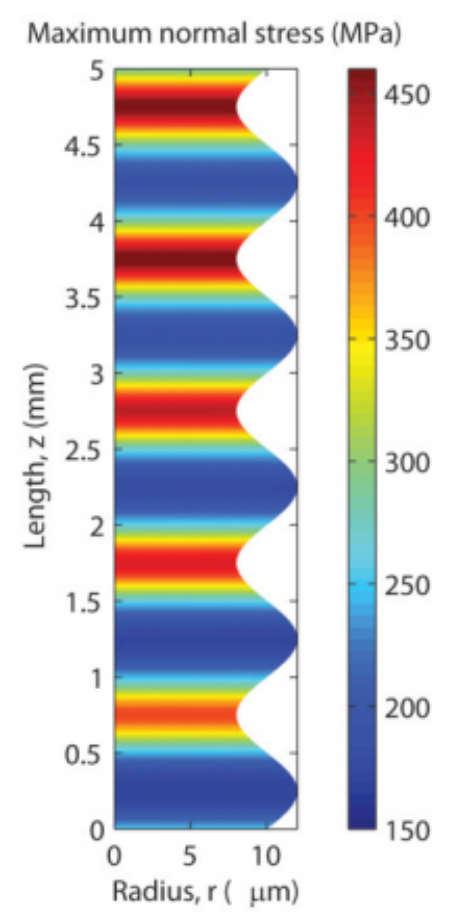

(c)

Maximum normal stress (MPa)

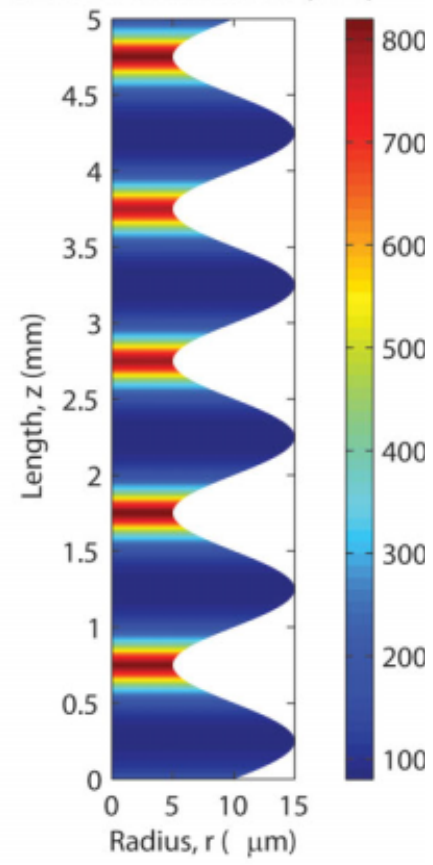

(f)

Figure 4 Contour plots of the maximum normal stress in the fibers with $n=5, v_{0}=20 \mathrm{~m} / \mathrm{s}$ and different $\delta_{r}$ : (a) $\delta_{r}=0$; (b) $\delta_{r}=1 \mu \mathrm{m}$; (c) $\delta_{r}=2 \mu \mathrm{m}$; (d) $\delta_{r}=3 \mu \mathrm{m}$; (e) $\delta_{r}=4 \mu \mathrm{m}$; (f) $\delta_{r}=5 \mu \mathrm{m}$. [Color figure can be viewed in the online issue, which is available at www.interscience.wiley.com.]

maximum value did not occur at the same narrowest site for different irregular fibers. For example, it occurred at the first narrowest site from the bottom for $\delta_{r}=1 \mu \mathrm{m}(\mathrm{b})$ at the last narrowest site for $\delta_{r}=3 \mu \mathrm{m}$ (d). This difference could be caused by the difference of the geometry of the fibers, which results in the difference in stress wave propagation and interference. 


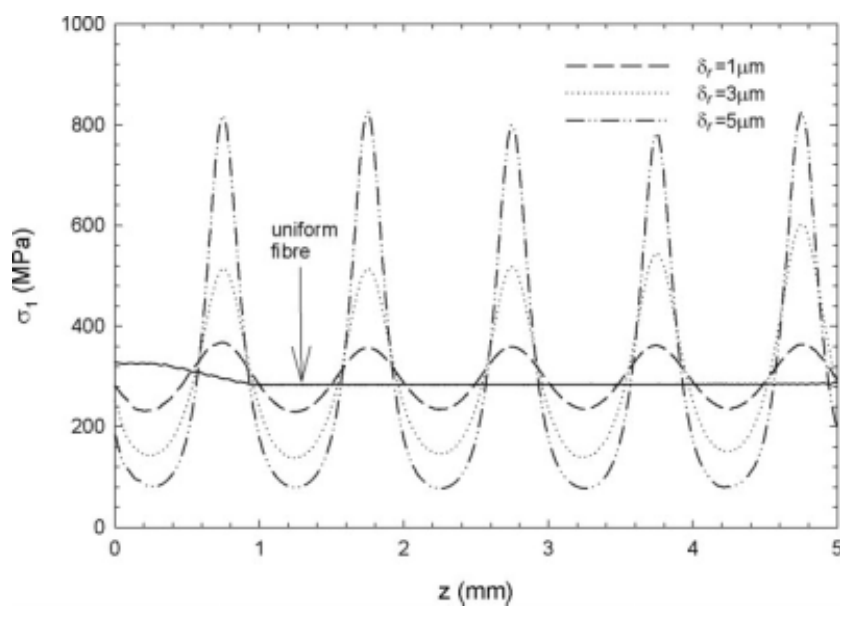

Figure 5 Variation of the first principal stress along the axial direction $z$ for irregular fibers with the same irregular frequency $n=5$ but different irregular radius amplitude $\delta_{r}$ under the same pulling speed $v_{0}=20 \mathrm{~m} / \mathrm{s}$, compared with the results of the regular uniform fiber.

2. All the contour plots show that the first principal stress mainly varies along the longitudinal direction. Its change in radial direction is negligibly small. This is due to the fact that the fiber is very thin (the radius is only $10 \mu \mathrm{m}$ ) and the length to mean radius ratio is very large (500). Therefore, we can use the curves of the principal stress versus the coordinate $z$ to better quantify the variation of the maximum normal stress. Figure 5 shows that for the uniform fiber, the largest value appears at the bottom and it is almost constant for $z>1 \mathrm{~mm}$. Unlike the uniform fiber, the stress in the irregular fibers varies wavily following the irregular shape of the fibers. Peak stress values appear at the narrowest sites and low-stress values appear at the widest sites.

3. The maximum first principal stress $\sigma_{1}^{\max }$ increases with the irregular radius amplitude $\delta_{r}$ as demonstrated in Figure 6(a). Under the given $v_{0}=20 \mathrm{~m} / \mathrm{s}$ and $n=5$, the value of $\sigma_{1}^{\max }$ increases from 328 to $826 \mathrm{MPa}$ from the uniform fiber to the irregular fiber with $\delta_{r}=5 \mu \mathrm{m}$.

For a given fiber, the influence of the pull speed $v_{0}$ on the maximum first principal stress is shown in Figure 6(a). All the four curves indicate that $\sigma_{1}^{\max }$ increases rapidly with the irregular radius amplitude $\delta_{r}$. Further more, we can see that, in general, $\sigma_{1}^{\max }$ increases with $v_{0}$ for a given fiber. For example, $\sigma_{1}^{\max }$ increased from 635 to $688 \mathrm{MPa}$ for the fiber $\delta_{r}=4$ $\mu \mathrm{m}$ and $n=5$. However, the four curves in Figure 6(a) are very close to each other. In comparison with the irregularity parameter $\delta_{r}$, the effect of pull speed on the maximum first principal stress seems to be not significant. Cautions should be taken to interpret these results. First, if the pull speed is extremely high, a significant high stress can be obtained. For example, calculation indicates that $\sigma_{1}^{\max }=1001 \mathrm{Mpa}$ when $v_{0}=40 \mathrm{~m} / \mathrm{s}$ for a fiber with $\delta_{r}=5$ and $n=5$, whereas $\sigma_{1}^{\max }=826 \mathrm{MPa}$ when $v_{0}=20 \mathrm{~m} / \mathrm{s}$ for the same fiber.

Second, the material properties are the same for different pull speeds in Figure 6(a). Physically, the elastic Young's modulus of fiber materials might increase with the pull speed. A figure published by Lynos indicates that the Young's modulus of polyethylene fibers increased by $200 \%$ when the nominal strain rate increased from $0.1 / \mathrm{min}$ (quasi-static state) to $2 \times 10^{7} / \mathrm{min}$ (which corresponds to the pull speed of $17 \mathrm{~m} / \mathrm{s}$ in our case). The sensitivity of the Young's modulus on the strain rate depends on the fiber type. In our current study, the irregular fibers correspond to natural fiber materials, such as wool fibers. To our knowledge, no published data on the sensitivity of the Young's modulus on the strain rate
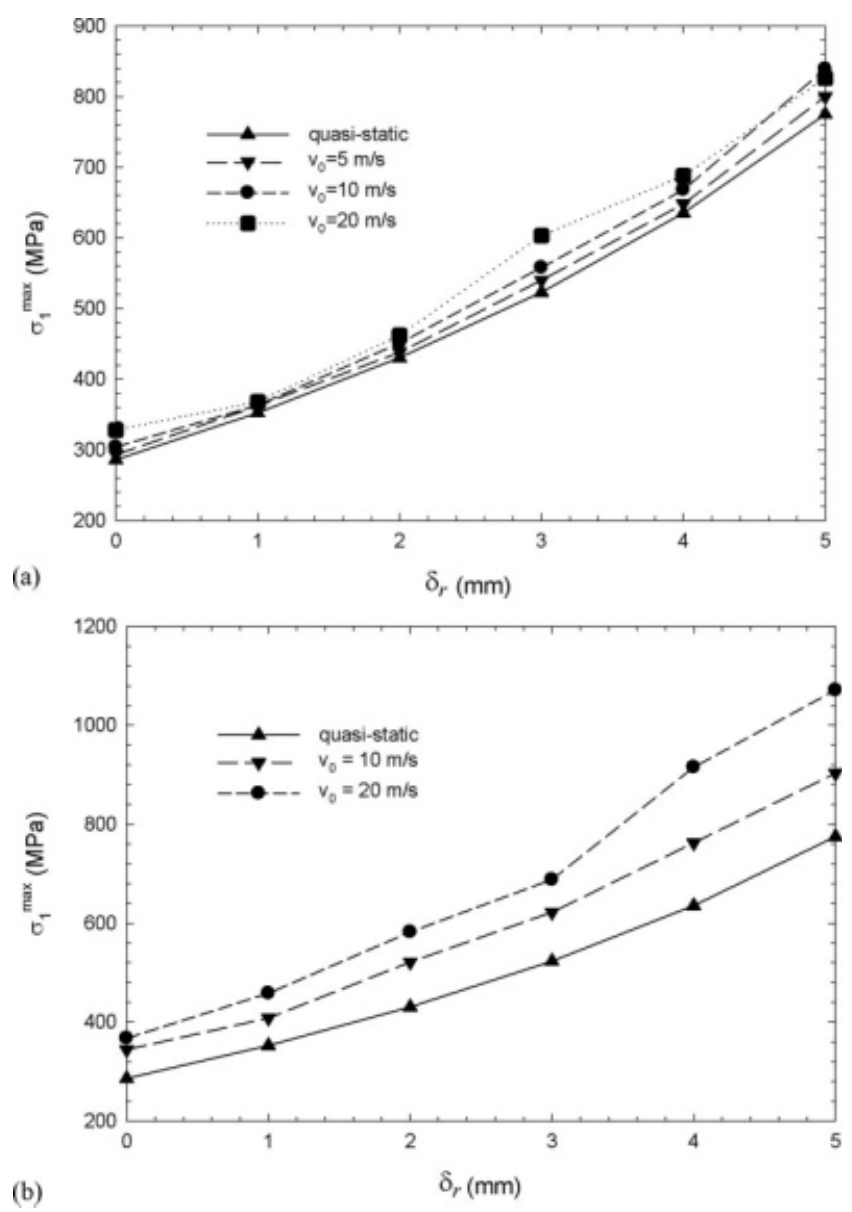

Figure 6 (a) The relationship between the maximum first principal stress $\sigma_{1}^{\max }$ and the irregular radius amplitude $\delta_{r}$ for $n=5$ under different pulling speeds with constant Young's modulus. (b) The relationship between the maximum first principal stress $\sigma_{1}^{\max }$ and the irregular radius amplitude $\delta_{r}$ for $n=5$ under different pulling speeds with the Young's modulus increasing with the pull speed. 


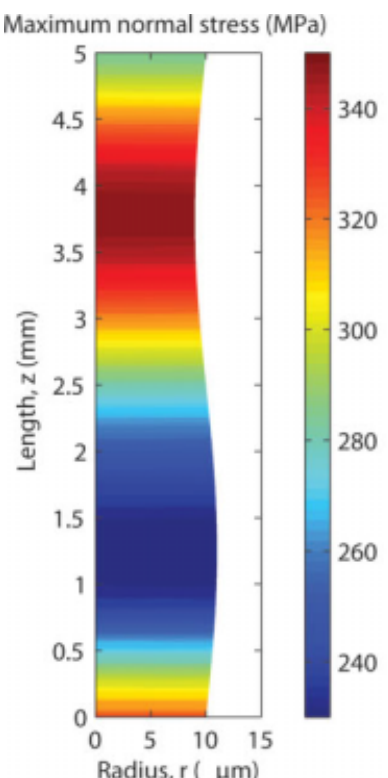

(a)

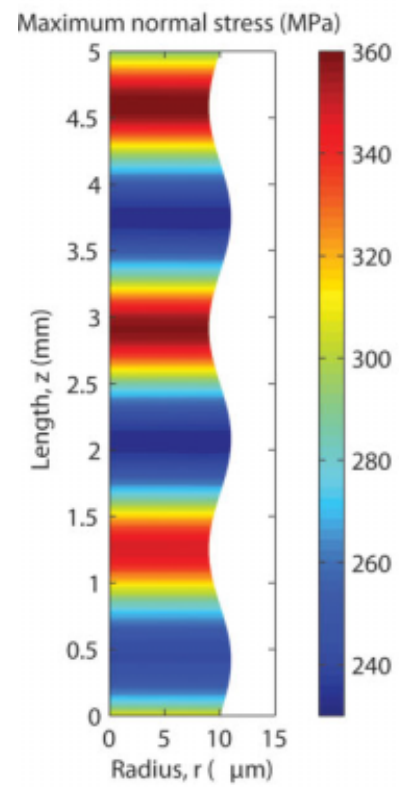

(c)

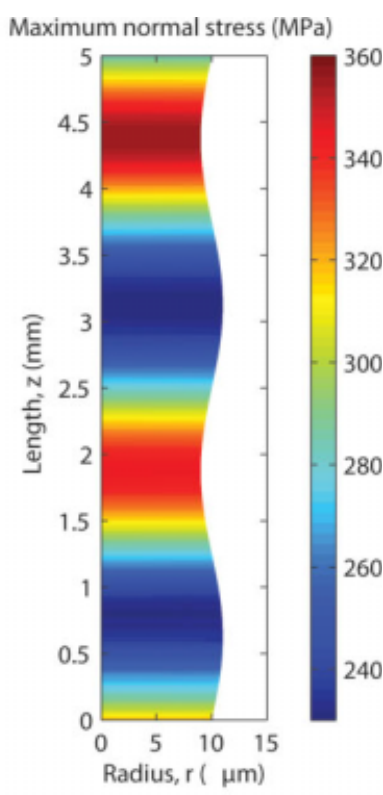

(b)

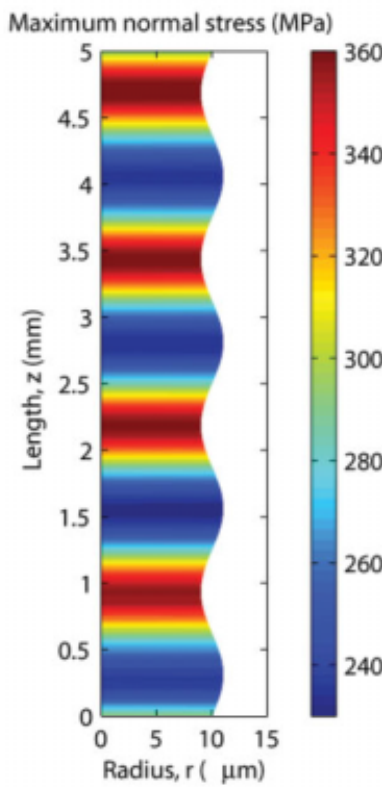

(d)

Figure 7 Contour plots of the maximum normal stress for fibers under $v_{0}=20 \mathrm{~m} / \mathrm{s}$ with $\delta_{r}=1 \mu \mathrm{m}$ and different $n$ : (a) $n=1$; (b) $n=2$; (c) $n=3$; and (d) $n=4$. [Color figure can be viewed in the online issue, which is available at www.interscience.wiley.com.]

for wool fibers is available. To examine the effect of the variation of Young's modulus with the strain rate, we chose $E=3750 \mathrm{MPa}$ for $v_{0}=20 \mathrm{~m} / \mathrm{s}, E=$ $3375 \mathrm{MPa}$ for $v_{0}=10 \mathrm{~m} / \mathrm{s}$ and kept $E=3000 \mathrm{MPa}$ for the quasi-static state based on the consideration that the Young's modulus of wool fiber is less sensitive to the strain rate than the polyethylene, and simulated the tensile tests again. As we can expect, the stress value presented in Figure 6(b) increases significantly with pull speed for a given fiber largely

due the increase in the Young's modulus. For example, $\sigma_{1}^{\max }$ increased from 903 to $1071 \mathrm{MPa}(18.6 \%$ increase) when $v_{0}$ changes from 10 to $20 \mathrm{~m} / \mathrm{s}$ for the fiber with $\delta_{r}=5 \mu \mathrm{m}$ and $n=5$. Reliable experimental data are required to further investigate the influence of the variation of the Young's modulus with the strain rate. In the current study, we mainly focused on the influence of the geometry irregularity. In the following simulations, we continued to use the constant Young's modulus for different pull speeds.

\section{Effects of irregular frequency $n$}

Under the same pulling speed of $v_{0}=20 \mathrm{~m} / \mathrm{s}$ and the same irregularity amplitude of $\delta_{r}=1 \mu \mathrm{m}$, Figure 7 shows the contour plots of the first principal stress in the fibers with different values of irregular frequency $n$. Again, the maximum first principal stress $\sigma_{1}^{\max }$ appears at the narrowest sites. However, this value does not change much from $n=1$ to $n=4$.

The effects of the irregular frequency $n$ on the maximum first principal stress $\sigma_{1}^{\max }$ are shown in
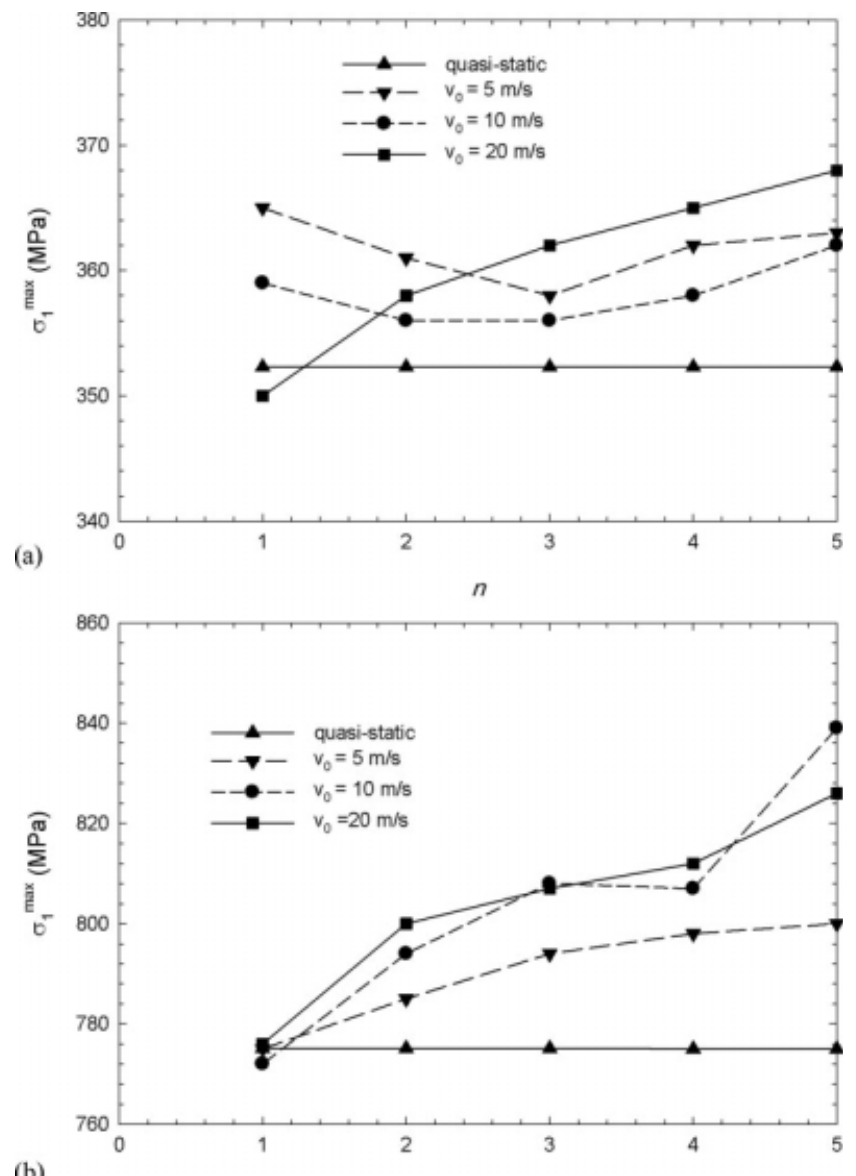

(b)

Figure 8 The relationship between the maximum first principal stress $\sigma_{1}^{\max }$ and the irregular frequency $n$ under different pulling speeds for (a) $\delta_{r}=1 \mu \mathrm{m}$; (b) $\delta_{r}=5 \mu \mathrm{m}$. 
Figure 8. Figure 8(a) shows the variation of $\sigma_{1}^{\max }$ when $n$ changes from 1 to 5 for fibers with the same value of $\delta_{r}=0.1 \mu \mathrm{m}$ under different pulling speeds. Under the quasi-static loading condition, $v_{0}=0$, $\sigma_{1}^{\max }$ has the same value of $352 \mathrm{MPa}$ for different values of $n$. However, under the dynamic loading condition $\left(v_{0} \neq 0\right)$, the irregularity frequency $n$ affects the maximum first principal stress $\sigma_{1}^{\max }$ as shown in Figure 8 . The $\sigma_{1}^{\max }$ varies marginally with the change in the value of $n$. For example, when the irregularity frequency $n$ changes from 1 to $5, \sigma_{1}^{\max }$ increases from 350 to $368 \mathrm{MPa}$ under the pulling speed $v_{0}=20 \mathrm{~m} / \mathrm{s}$. The increase is about $4.6 \%$. Under the other two loading conditions of $v_{0}=5$ $\mathrm{m} / \mathrm{s}$ and $v_{0}=10 \mathrm{~m} / \mathrm{s}, \sigma_{1}^{\max }$ decreases slightly and then increases slightly when $n$ increases from 1 to 5 . Overall, one can conclude that the influence of the irregularity frequency $n$ on the maximum first principal stress $\sigma_{1}^{\max }$ is not significant. Now the reference $\sigma_{1}^{\max }$ value, corresponding to quasi-static loading, is $775 \mathrm{MPa}$ in Figure 8(b), which is due to the large value of $\delta_{r}=5 \mu \mathrm{m}$. The difference compared with Figure $8(a)$ is that $\sigma_{1}^{\max }$ increases slightly with $n$, but the increasing percentage is still very small.

\section{CONCLUSIONS}

The dynamic responses of irregular wool fibers under axial tension have been investigated through FE simulations. The irregularity is represented by the sinusoidal shape profile along the fiber axis. Considering the fiber breakage problem in fiber processing, the maximum first principal stress due to the dynamic pulling has been examined. We conclude:

1. The first principal stress mainly varies along the longitudinal direction. Its change in radial direction is negligibly small.
2. The maximum first principal stress in an irregular fiber always appears in one of the narrowest crosssections, which is the weakest link of the fiber.

3. The maximum first principal stress is very sensitive to the change in the irregularity amplitude. The stress value increases dramatically with the increase in the irregularity amplitude.

4. The irregularity frequency has a limited effect on the maximum first principal stress.

The authors are grateful to the two reviewers for their constructive comments for improving the article.

\section{References}

1. Harrowfield, B.; Plate, D.; Eley, J. Wool Scouring and Worsted Carding: New Approaches, CSIRO Symposium; Geelong, 1986; p 33.

2. Peirce, F. T. J Text Inst 1926, 17, T355.

3. Banky, E. C.; Slen, S. B. Text Res J 1955, 25, 358

4. Kenny, P.; Chaikin, M. J Text Inst 1959, 50, T18.

5. Collins, J. D.; Chaikin, M. Text Res J 1965, 35, 679.

6. Collins, J. D.; Chaikin, M. J Text Inst 1966, 57, T45.

7. Collins, J. D.; Chaikin, M. Text Res J 1969, 39, 121.

8. He, W. Y.; Wang, X. G.; Zhang, S. Text Res J 2001, 71, 556.

9. Zhang, Y. P.; Wang, X. G. Wool Technol Sheep Breed 2000, 48, 303.

10. Deng, C. M.; Wang, L. J.; Wang, X. G. Fiber Polym 2007, 8, 289.

11. Lyons, W. J. Impact Phenomena in Textiles; MIT Press: Cambridge, MA, 1963.

12. Reis, P. J. Aust J Agr Res 1992, 43, 1337.

13. Postle, R.; Carnaby, G. A.; de Jong, S. The Mechanics of Wool Structures; Wiley: New York, 1988.

14. Hibbitt, D.; Karlsson, B.; Sorensen, P. ABAQUS version 6.5; ABAQUS: Providence, RI, 2005.

15. Love, A. E. H. A Treatise on the Mathematical Theory of Elasticity; Dover Publications: New York, 1994.

16. Meyers, M. A. Dynamic Behaviour of Materials; Wiley: New York, 1994.

17. Danzer, R.; Fischer, F. D.; Yan, W. J Eur Cer Soc 2000, 20, 901. 